\title{
Random forest-based imputation outperforms other methods for imputing LC-MS metabolomics data: a comparative study
}

Marietta Kokla ${ }^{1 *}$ (D, Jyrki Virtanen ${ }^{1}$, Marjukka Kolehmainen ${ }^{1,2}$, Jussi Paananen ${ }^{3 \dagger}$ and Kati Hanhineva ${ }^{1 \dagger}$

\begin{abstract}
Background: LC-MS technology makes it possible to measure the relative abundance of numerous molecular features of a sample in single analysis. However, especially non-targeted metabolite profiling approaches generate vast arrays of data that are prone to aberrations such as missing values. No matter the reason for the missing values in the data, coherent and complete data matrix is always a pre-requisite for accurate and reliable statistical analysis. Therefore, there is a need for proper imputation strategies that account for the missingness and reduce the bias in the statistical analysis.

Results: Here we present our results after evaluating nine imputation methods in four different percentages of missing values of different origin. The performance of each imputation method was analyzed by Normalized Root Mean Squared Error (NRMSE). We demonstrated that random forest (RF) had the lowest NRMSE in the estimation of missing values for Missing at Random (MAR) and Missing Completely at Random (MCAR). In case of absent values due to Missing Not at Random (MNAR), the left truncated data was best imputed with minimum value imputation. We also tested the different imputation methods for datasets containing missing data of various origin, and RF was the most accurate method in all cases. The results were obtained by repeating the evaluation process 100 times with the use of metabolomics datasets where the missing values were introduced to represent absent data of different origin.
\end{abstract}

Conclusion: Type and rate of missingness affects the performance and suitability of imputation methods. RF-based imputation method performs best in most of the tested scenarios, including combinations of different types and rates of missingness. Therefore, we recommend using random forest-based imputation for imputing missing metabolomics data, and especially in situations where the types of missingness are not known in advance.

Keywords: Metabolomics, Imputation, Missing values, High dimensional data, RF, MAR, MNAR, MCAR

\section{Background}

Metabolomics studies involve investigation of small molecules or molecular features, which are end results of cellular metabolism in biological samples. The primary motivation behind the technology is to profile as many molecular features as possible with the chosen instrumental set-up and thereafter study e.g. alterations

\footnotetext{
* Correspondence: marietta.kokla@ueffi

†Jussi Paananen and Kati Hanhineva contributed equally to this work.

'Institute of Public Health and Clinical Nutrition, University of Eastern Finland,

Kuopio Campus, P.O. Box 1627, Fl-70211 Kuopio, Finland

Full list of author information is available at the end of the article
}

in metabolism under different conditions. One of the most commonly used technologies in metabolomics is liquid chromatography combined to mass spectrometry (LC-MS) which generates a vast amount of multivariate data amenable for multiple biostatistical, bioinformatical and any other computational data-analytical approaches $[1,2]$.

A pre-requisite for reliable data-analysis in metabolomics experiment is that the quality of the data is monitored efficiently. One of the main drawbacks in LC-MS metabolomics data is that it typically may contain a large

(c) The Author(s). 2019 Open Access This article is distributed under the terms of the Creative Commons Attribution 4.0 International License (http://creativecommons.org/licenses/by/4.0/), which permits unrestricted use, distribution, and reproduction in any medium, provided you give appropriate credit to the original author(s) and the source, provide a link to the Creative Commons license, and indicate if changes were made. The Creative Commons Public Domain Dedication waiver (http://creativecommons.org/publicdomain/zero/1.0/) applies to the data made available in this article, unless otherwise stated. 
proportion of missing values, even in the range of 30$50 \%[3,4]$. The procedure of treating missing values is called imputation, and it focuses on replacing the missing data with values using the information that is available from the existing data. So far, several methods have been introduced for the imputation of metabolomics data $[3,5-7]$.

The missingness of data is generally due to either true absence of the compound in the measured sample, or the molecular feature can be present in the sample at a concentration below the detection limit of the mass spectrometer. However, in many cases the missing data value results from inappropriate transformation of the measured MS signal to the numerical data format. The raw data processing involves various steps including peak detection, peak alignment, adduct/neutral loss detection, baseline correction and noise reduction. Therefore it is one of the most challenging computational processes in the metabolomics experiment and thus prone to errors [8]. Thus, the related statistical analysis and the interpretation of metabolomics data will be biased, in case the missing values are not treated properly.

Given the magnitude of missing data and the ambiguity that surrounds the statistical analysis of metabolomics datasets, it is desirable first to investigate and then consider the properties of missing data prior to applying any imputation methods. Rubin and Little have established the foundations of missing data theory $[9,10]$ and according to them missing data can be classified into three missing mechanisms based on the nature of the absence of values from the data matrix: Missing Completely at Random (MCAR), Missing at Random (MAR) and Missing Not at Random (MNAR). The first mechanism, MCAR, describes the process that the missing values cannot be attributed neither to the molecular features that are present or to the molecular features that are missing in the samples. This mechanism is characterized by a randomness in the occurrence of missing data, which could also be accounted as a zero correlation between the missing and the observed part in the data. In the MAR mechanism, we observe a systematic correlation between the missing molecular features and the observed data, but not with the missing data itself, meaning that the probability of a molecular feature being missing is determined by other observed molecular features. Lastly, MNAR is the most troublesome mechanism, because it depends on the unobserved part of the data, when missing values are neither MCAR nor MAR. MNAR denotes the process that the value of the molecular feature is causal for missingness and that is why it is missing, and this type of missing data is usually characterized in many metabolomics studies as left truncated data (molecular features occur below the detection limit) [11].
Furthermore, diagnosing what type of mechanisms of missingness there are in a dataset is challenging, and there is not yet a solid straightforward method to diagnose these mechanisms [12, 13]. Many studies have compared imputation strategies such as different variations of K-nearest neighbors or used machine learning techniques to replace missing values, and investigated how they can alter the biological information within simulated or real metabolomics datasets $[2,5,6,11,14-18]$.

In order to obtain reliable metabolomics data, it is necessary to find the most suitable method or approach for treating the missing values of various origin. Hence, we performed a detailed evaluation on the performance of various imputation methods on sub-sets of data generated from human plasma samples measured with LC-MS based non-targeted metabolite profiling analysis. In this work, missing data was simulated with the three main missing mechanisms (MCAR, MAR, MNAR) and four additional combined mechanisms (MCAR-MAR, MCAR-MNAR, MAR-MNAR, MCAR-MAR-MNAR) which we created in order to introduce a more realistic missing data structures. The datasets containing missing values based on the above seven different missing mechanisms were imputed with nine commonly applied imputation methods in metabolomics: ZERO, MEAN, minimum value (MIN), half minimum ( $1 / 2$ MIN), Singular Value Decomposition (SVD), Probabilistic Principal Component Analysis (PPCA), Bayesian Principal Component Analysis (BPCA), Random Forest (RF), and K-Nearest Neighbors (KNN).

\section{Results}

In order to test the performance of the nine different imputation methods, we generated sub-datasets from 12 LC-MS metabolomics datasets (Fig. 1), and simulated missing values to these according to seven different missing mechanisms; MCAR, MAR, MNAR, MCARMAR, MCAR-MNAR, MAR-MNAR, and MCAR-MARMNAR, in four different proportions of missing values (5, 10, 20 and 30\%). The imputation process was repeated 100 times as visualized in the workflow chart in Fig. 2. Furthermore, in order to avoid potentially biased comparisons, we have explored the performance of KNN method by optimizing the parameter settings to reach optimal performance. The number of neighbors $K$ was chosen to be equal with 10 . In the RF, method there is no need for tuning the parameters nor does it require assumptions about distributional aspects of the data as suggested [19] so we chose the default values of the missForest function; maximum iterations was set to 10 and the number of trees was chosen to be 100 .

The tested imputation methods were ZERO, MEAN, MIN, $1 \frac{1}{2}$ MIN, SVD, PPCA, BPCA, RF, and KNN. Results from the performances of all the imputation methods are illustrated in Fig. 3 as heatmaps. The heatmaps show 
a) KIHD study (258 samples)

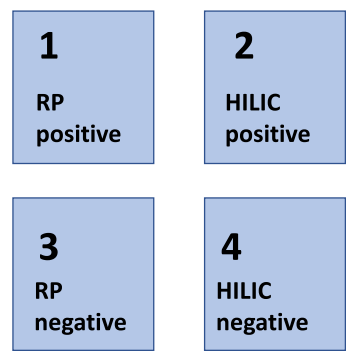

b) BS study (47 samples)

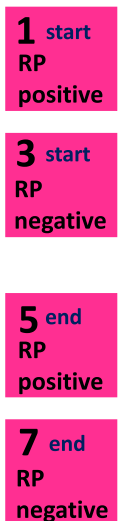

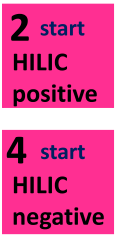

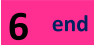

HILIC

positive

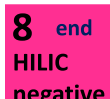

Fig. 1 Description of the datasets. Datasets from two different studies were used; KIHD (Kuopio Ischaemic Heart Disease Risk Factor Study) and BS (Berry Study).The data were analyzed with LC-MS technology and each analytical chromatographic mode is consider a separate dataset. In total 12 datasets were used $\mathbf{a}$ four datasets from the KIHD study and $\mathbf{b}$ eight datasets from the BS intervention study (four chromatographic modes per time point)

the average Normalized Root Mean Square Error (NRMSE), which calculates the difference in the estimation between the imputed value and the original value for every molecular feature that contains missing values, after 100 permutations. Each heatmap reflects one missing mechanism and the different shades of blue are associated with the different values of NRMSE that indicate the performance of each imputation method in four different percentages of missing data.

\section{Comparison of imputation methods based on heatmaps} In the MCAR case, RF and KNN performed the best by having the smallest NRMSEs, while PPCA, BPCA and MEAN, even though having similar performances with

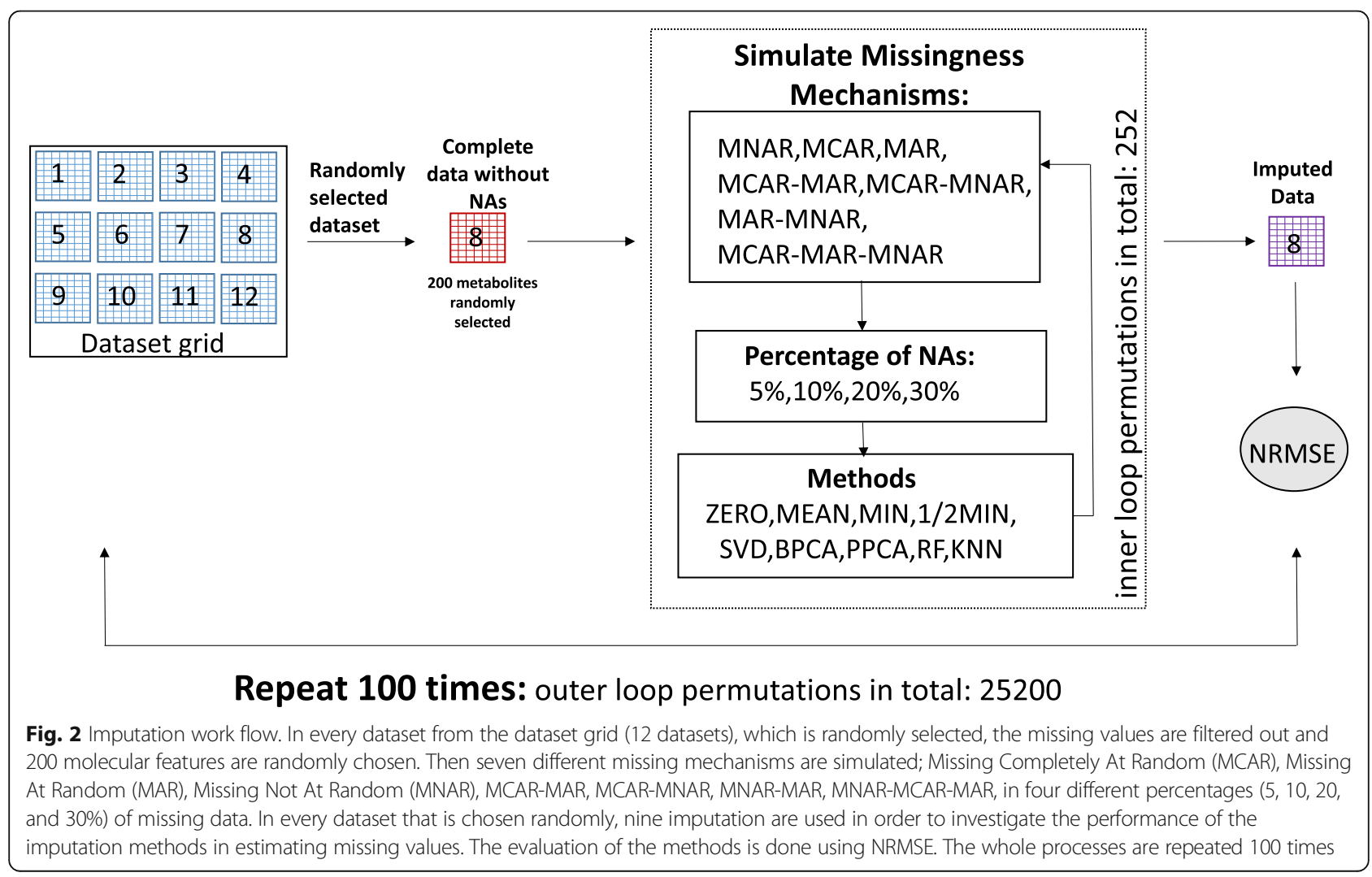




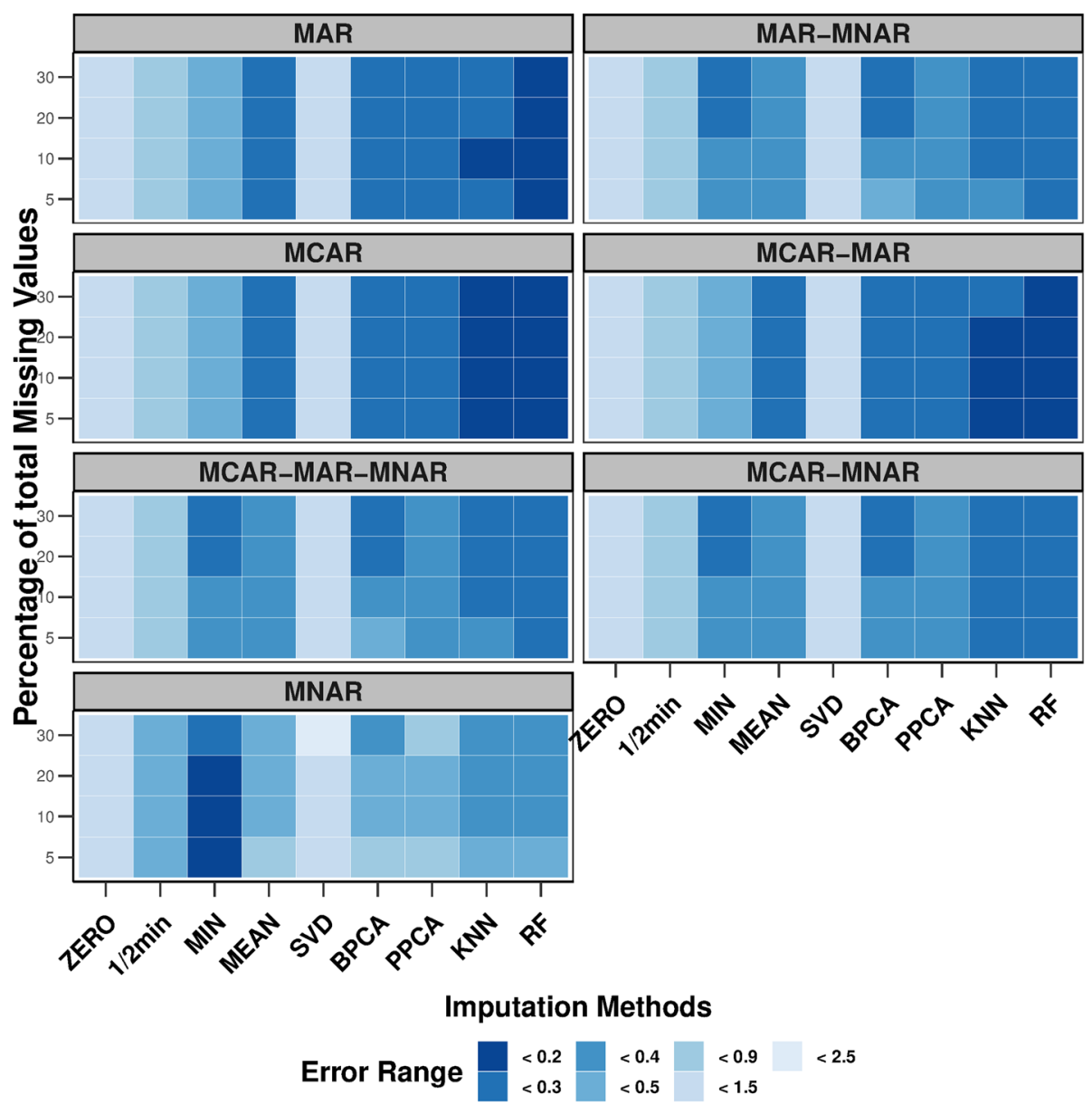

Fig. 3 Heatmaps. Heat maps representing the average performance of nine imputation methods after 100 permutations; zero (ZERO), 1/2 -minimum (1 $12 \mathrm{M}$ MIN), minimum (MIN), Random forest (RF), mean (MEAN), K-nearest neighbor (KNN), Bayesian Principal Component Analysis (BPCA), Probabilistic Principal Component Analysis (PPCA), Singular Value Decomposition (SVD) in seven missing mechanisms (each box); MCAR, MAR, MNAR, MCAR-MAR, MCAR-MNAR, MAR-MNAR, MCAR-MAR-MNAR. The darker blue color indicates that the error is small whereas, when the color transforms to lighter shades, this is an indication that the error becomes higher by the corresponding imputation method

each other, could not outperform RF and KNN. SVD was the method with the highest NRMSEs compared to the other methods. ZERO imputation, $1 / 2$ MIN and MIN method had smaller NRMSEs compared to the SVD but still higher than RF and KNN.

The MAR missing mechanism had relatively similar results as the MCAR missing mechanism, with RF being the method with significantly lower NRMSEs than the others. The NRMSEs of the KNN were higher than the ones coming from RF when $5 \%$ of the data were missing. When $10 \%$ of the data were missing, KNN performs the same as RF, however, for higher percentages of missing values the error increased again. In the case of the other imputation methods, the trend of the performances were similar to the results obtained for the MCAR case.

In the MNAR missing mechanism, MIN was the best performing method compared to the other ones. It is interesting to note that when the number of missing values increases and starts reaching 30\%, the NRMSE of the MIN also increases. RF and KNN had similar results with each other and achieved their best smallest NRMSEs between 10 to $30 \%$ proportion of the missing values. ZERO and SVD were consistent in having high errors for all the percentages of missing values compared to the other methods. Furthermore, MIN was more robust than $1 / 2$ MIN with smaller NRMSEs for all the percentages of missing values. PPCA, BPCA and MEAN started with relative high NRMSEs when $5 \%$ of the data were missing compared to the MIN imputation, but the NRMSEs decreased, although not significantly, when the range of missingness was between 10 to $20 \%$. When the missingness reached $30 \%$, the NRMSE of BPCA and MEAN continued to drop but for PPCA method it increased again.

For the mixed missingness MCAR-MAR, RF and KNN had the smallest NRMSE. RF's NRMSE was constant for 
all the percentages of missing values in comparison with $\mathrm{KNN}$, errors of which increased when the range of missing values reached 30\%. BPCA, PPCA, and MEAN had similar results for all the four percentages of missing values. ZERO and SVD had similar performances with high NRMSEs in all percentages. Between MIN and $1 / 2$ MIN methods, MIN value had the smallest NRMSEs but still higher than RF, KNN, BPCA, PPCA and MEAN.

With MAR-MNAR, RF performed the best in all the missing percentages. KNN performed as good as RF, with a small increase of the error when $5 \%$ of the data were missing. MIN together with BPCA had similar performances with KNN and RF, when the percentages of missing values were between 20 and $30 \%$, but for smaller percentages of missing values (between 5 and 10\%) they had relatively high NRMSEs compared to RF. The rest of the methods as an overall performance had higher NRMSEs compare to RF.

By combining MCAR and MNAR mechanisms together (MCAR-MNAR) we had similar performances in the imputation methods as in the MAR-MNAR case. Once again KNN and RF were the ones with the smallest errors. The only difference was that at the 5\% range of missing values, KNN and BPCA had smaller NRMSEs compared to the MAR-MNAR case.

Lastly, when we had three missing value mechanisms mixed together (MCAR-MAR-MNAR) the results looked similar as with the MAR-MNAR missing mechanism. The best performing method was RF followed by KNN.

\section{Summary results}

Figure 4 shows the summary results from six out of nine imputation methods that we evaluated; MIN, MEAN,
BPCA, PPCA, KNN, and RF. This choice was based on the performances of the methods illustrated in the heatmaps in Fig. 3. For more details two summary tables (Tables S1-S2) containing the mean, standard deviation of the average NRMSEs of every imputation method for all the four percentages of missing values have been added in the Additional file 1. As suggested by these results, the most troublesome missingness is the MNAR with the MIN imputation is the best approach by having the smallest NRMSE. The rest of the five methods (MEAN, BPCA, PPCA, KNN, and RF) have high NRMSEs and also standard deviations. A relatively high error bar suggests that the method in question is not stable when imputing missing values and for each permutation provides different estimates. For the MCAR and MAR mechanism the conclusions are the same as in the heatmaps, with RF being the most appropriate choice. Furthermore, for the remaining four kinds of mixed missing mechanisms (MCAR-MAR, MCARMNAR, MAR-MNAR, and MCAR-MAR-MNAR) the performances are similar with MCAR and MAR cases, with RF being the best imputation method followed by KNN.

\section{Discussion}

Here we tested nine imputation methods on seven different missing mechanisms with twelve metabolomics datasets. Our results indicated that RF was the most effective means for imputing metabolomics datasets based on the lowest NRMSE value and error bars in nearly all the simulated datasets representing different combinations of missing values. RF is a powerful method, even though the whole imputation procedure with RF has a computation expense due to the intensive

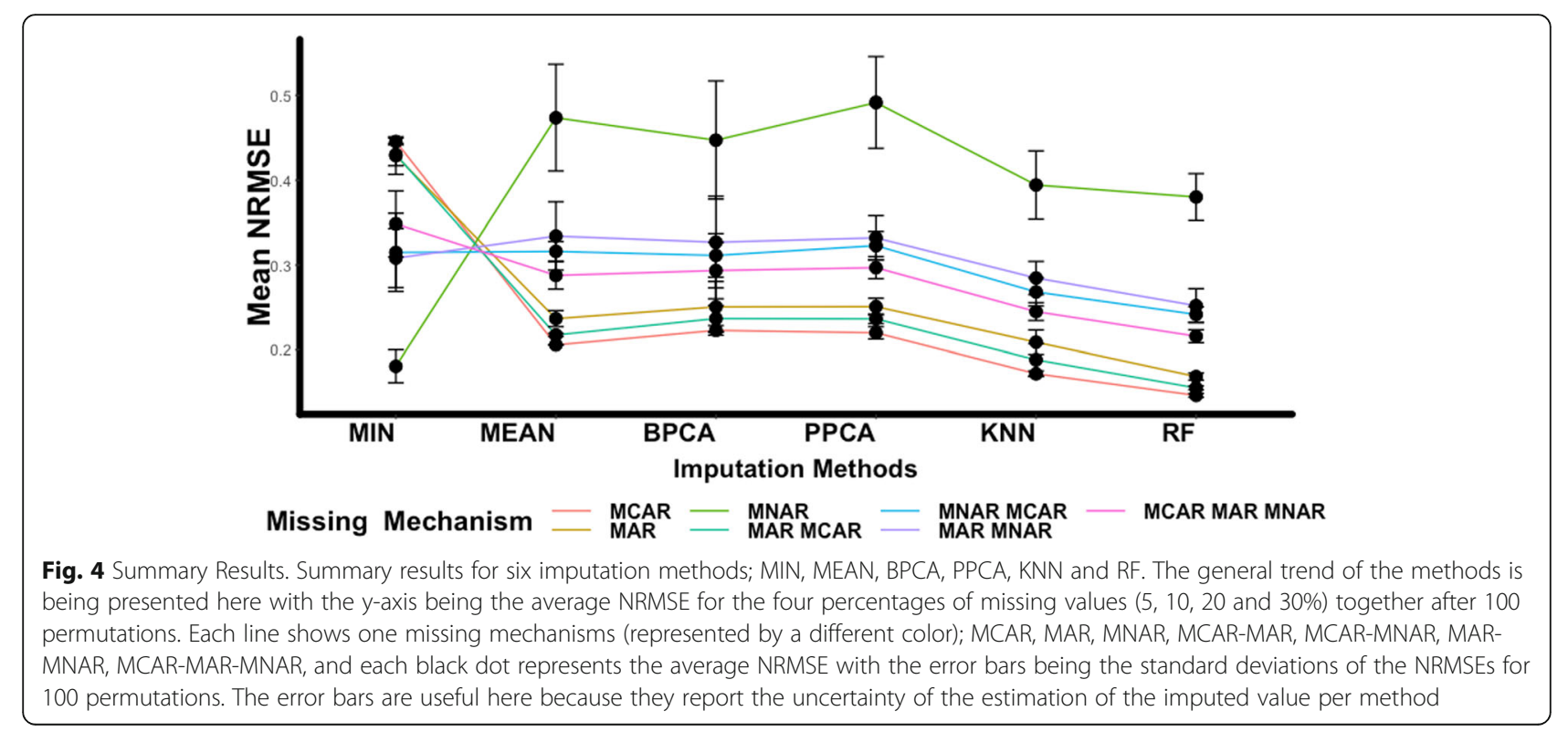


iterations (Additional file 1: Figure S7), it is well known that it can handle both parametric and non-parametric data sets of complex linear and non-linear problems and there is no need to perform preprocessing at the data beforehand. On the other hand SVD where is a popular approach for data analysis and data processing (e.g., dimension reduction), PPCA which is basically PCA with an expectation- maximization (EM) approach and BPCA which is based on three processes, including principal component $(\mathrm{PC})$ regression, Bayesian estimation, and an EM-like repetitive algorithm, they perform better if the data are being transformed first before imputation [2]. In our experimental setting we didn't consider any preprocessing methods, even when this is common in other imputation studies, because in most metabolomics studies, almost half of the molecular features do not follow the normal distribution as their values are highly positively or negatively skewed. When a molecular feature is Log-transformed in order to meet the normality assumptions before imputing, we are not only changing the distribution of that particular molecular feature but also we disturb the relationships between that molecular feature with the rest of the data, and therefore, this approach can lead in imputing outliers or/and creating more bias than just imputing the skewed molecular feature.

One aspect of our results that was not aligned with the findings from another study [20], was the very poor performance of the SVD imputation method. SVD begins the imputation procedure by replacing all missing values with zero values and then iterates through singular value decompositions until convergence. This procedure may create effects on the data structure that can alter the factorization of the data matrices. In theory that shouldn't happen because the errors in the prediction that are being introduced in the initialization step should be cancelled out by the matrix-factorization approach. On the other hand, BPCA and PPCA also rely on dimension reduction, performed better than SVD. This was because they include a probabilistic model that minimize the principal axes that are not relevant, and make these two methods more robust to changes in the data structure and in the cases of MCAR, MAR and MCAR -MAR.

We also observed that in the cases of MNAR, MCARMAR-MNAR, MAR-MNAR, MCAR-MNAR the performance of BPCA improved when the proportion of missing values increased and this phenomenon, which may seem at first contradictory, could be due to the fact that multivariate models perform better in a more uniformly distributed missingness across datasets.

The treatment of missing data by single value replacement, such as MIN, performs better when missing data arise from censoring below the detection limit, while other methods, such as RF, which is a method based on local structures, performs better when randomness is involved. KNN method performs slightly better than MEAN imputation which was expected since it is considered as an advancement over the MEAN imputation [17]. However, KNN showed instability in the prediction of missing values especially in the MAR case and this spurious performance is highlighting the fact that this method probably is not the most suitable for imputing such complex datasets.

In addition, it has been shown that certain methods favor more specific mechanisms than others, but there is no imputation method so far that works well for all three types of mechanisms [20]. By closely investigating the results presented in Figs. 3 and 4, it seems that RF is estimating missing values with the lowest prediction error. Its performance is consistent in the four percentages of missing values and for the majority of the missing mechanisms. The exception is the left-truncated MNAR, where the MIN performs better. These results coincide with other studies as well, that tested the performance of RF with other methods [2, 20].

However, in the case of the MNAR-MCAR-MAR, BPCA and MIN had similar performances with RF and $\mathrm{KNN}$, especially for higher percentages of missing values of 20 and $30 \%$. This result could indicate that as the number of missing values increases, the left truncation of missingness starts to affect the data while other kinds of missing mechanisms are also present. If the data are left truncated then the preferable option will be the MIN or any other imputation method, such as KNN-TN and GSimp, that perform well for this type of missingness as has been proposed also earlier [11, 20, 21].

In this work, the missing values were controlled and removed using one of the three earlier characterized missing mechanisms or the combination of those. However, most of the times in real life the reason for a value to be absent is unknown. In many studies different imputation strategies have been compared, or it has been investigated that how different imputation methods can alter the biological information within simulated or real metabolomics datasets $[2,16,18]$. The detection of the missing mechanism is not typically performed in metabolomics field [22] but some efforts have been done in other research areas focusing on simulated data only [13]. If the missing mechanism can be detected with high certainty, it could reduce the bias occurring from the improper choice of an imputation method.

One limitation that our study has, is in the way the current simulation process of missing values is carried out. We cannot fully control the missing patterns simulation as we indented. That means that even if intended to remove the data with the MAR mechanism we may create missing patterns by random chance that resemble the MNAR mechanism especially when the percentage 
of the missing values starts to increase. In order to reduce this effect, we use moderate sizes of missingness, no higher than $30 \%$. Higher percentages regardless the missing mechanism tends to create MNAR missing patterns.

In this study, also we did not include any information about the different groupings inside the imputation study. When selecting the subset of 200 molecular features from the metabolomics data, we were focusing on data that was present for all the measured samples, in order to be able to simulate the missingness. We do acknowledge that when addressing complete metabolomics dataset, the grouping needs to be taken into account to avoid bias, and even introducing error, and therefore the imputation needs to be potentially performed in a group-wise manner. However, this would require further development to the utilized methods.

\section{Conclusion}

Type and rate of missingness affects the performance and suitability of imputation methods. In conclusion, RF-based imputation method performs best in most of the tested scenarios, including combinations of different types and rates of missingness. Therefore, we recommend using RF-based imputation for imputing missing metabolomics data, since typically the origin of missingness is not known in advance. In addition, our approach to evaluate the performance of imputation methods on metabolomics datasets is applicable also to other highdimensional data that contain missing values.

\section{Methods}

\section{Metabolomics data}

The metabolomics datasets used in the current study were obtained from two human based-nutritional studies carried out at the University of Eastern Finland. The Kuopio Ischaemic Heart Disease Risk Factor Study (KIHD) is an epidemiological study focusing on the effect of diet and lifestyle on cardiovascular disease risk in middle-aged men from eastern Finland [23]. A subset of 258 participants was randomly selected for a study focusing on the metabolic impact of egg consumption [24].

(https://www.uef.fi/web/nutritionepidemiologists/kuopio-ischaemic-heart-disease-risk-factor-stud-kihd-1984-).

The other dataset used in the current work was obtained from a human dietary intervention, namely the Berry Study (BS) $[25,26]$. BS was a controlled 16-week interventional trial, where plasma was collected from 47 individuals in two time periods; at the baseline (start of intervention) and at the 8-week follow up (end of intervention). Twelve individuals served as a control group, and the rest were divided into two experimental groups consuming two different types of berries.
The samples from these two studies were analyzed by UHPLC-qTOF-MS system (Agilent Technologies, Waldbronn, Karlsruhe, Germany) that consisted of a 1290 LC system, a Jetstream electrospray ionization (ESI) source, and a 6540 UHD accurate-mass qTOF spectrometer. The samples were analyzed using two different chromatographic modes, i.e. reversed phase (RP) and hydrophilic interaction (hilic) chromatography and the data were acquired in both positive $(+)$ and negative $(-)$ polarity. Here we consider each of these four analytical modes as separate datasets. The data pre-processing was carried out as described earlier [25, 27].

From the KIHD study we created four datasets (four analytical modes) and from the BS study, eight datasets representing the two time points and four analytical modes (Fig. 1). In order to create the datasets for the evaluation of the imputation methods, we considered molecular features that were present in all samples. Table 1 provides information about how many molecular features we had in each datasets and the total number of missing values. During the simulation processes, every time we repeated the simulation, we randomly picked one dataset out of the 12, and randomly selected 200 molecular features from this particular dataset that does not include any missing values, and this new subdataset was used to simulate missing values and test the imputation methods thereafter. Additional boxplots and correlation plots show the new complete sub-datasets in more detailed (Additional file 1: Figures S1-S6).

\section{Missing mechanisms simulation}

MCAR missingness was simulated by randomly removing values from the data set using the uniform distribution. Different rates of missingness were simulated by removing different proportions of the values $(5,10,20$, and $30 \%)$.

In the MAR missing mechanism, the missing values depend on the observed part of the data. In our simulation, we model a situation where a high abundance of a molecular feature $\mathrm{X}_{1}$ will lead to missingness of a molecular feature $X_{2}$ in the same sample. The simulation process begins by randomly choosing two different molecular features; $X_{1}$ and $X_{2}$. We sort the values of $X_{1}$ from minimum to maximum, and choose a cut-off percentage point randomly from chi-squared distribution, divide it by 30 and limit to range $0 . .1$. This approach will limit the cut-off percentage between 0 to $100 \%$, with mean of $3.3 \%$ and standard deviation of $4.7 \%$ (empirically simulated with 1 million values). This selected cutoff percentage will be used to set this proportion of highest $\mathrm{X}_{2}$ values to missing, simulating MAR missingness. After this, new $X_{1}$ and $X_{2}$ are randomly selected, and the procedure is repeated until total desired 
Table 1 Information about the total number of molecular features, number of missing values and total number of complete molecular features in each dataset

\begin{tabular}{|c|c|c|c|c|}
\hline Study & $\begin{array}{l}\text { LC-MS analytical } \\
\text { mode }\end{array}$ & $\begin{array}{l}\text { Total number of molecular } \\
\text { features }\end{array}$ & $\begin{array}{l}\text { Number of missing values per } \\
\text { dataset }\end{array}$ & $\begin{array}{l}\text { Number of molecular features without missing } \\
\text { values }\end{array}$ \\
\hline \multirow[t]{4}{*}{$\mathrm{KIHD}$} & Hillic positive & 2407 & 7519 & 204 \\
\hline & Hillic negative & 1228 & 64,256 & 299 \\
\hline & RP positive & 1228 & 24,451 & 509 \\
\hline & RP negative & 2407 & 95,079 & 498 \\
\hline \multicolumn{5}{|l|}{ BS } \\
\hline $\operatorname{Start}\left(\mathrm{T}_{0}\right)$ & Hillic positive & 1290 & 7140 & 518 \\
\hline $\begin{array}{l}\text { End } \\
\left(T_{1}\right)\end{array}$ & Hillic positive & 1290 & 7131 & 508 \\
\hline $\operatorname{Start}\left(T_{0}\right)$ & Hillic negative & 725 & 2701 & 383 \\
\hline $\begin{array}{l}\text { End } \\
\left(T_{1}\right)\end{array}$ & Hillic negative & 725 & 2766 & 383 \\
\hline $\operatorname{Start}\left(\mathrm{T}_{0}\right)$ & RP positive & 1177 & 11,679 & 1066 \\
\hline $\begin{array}{l}\text { End } \\
\left(T_{1}\right)\end{array}$ & RP positive & 1177 & 11,526 & 1152 \\
\hline $\operatorname{Start}\left(\mathrm{T}_{0}\right)$ & RP negative & 2286 & 4643 & 578 \\
\hline $\begin{array}{l}\text { End } \\
\left(T_{1}\right)\end{array}$ & RP negative & 2286 & 5146 & 559 \\
\hline
\end{tabular}

proportion of total missingness $(5,10,20 \%$, or $30 \%)$ for the whole data set is reached.

The MNAR missing mechanism is very similar to the MAR simulation process. We chose randomly one molecular feature, $\mathrm{X}_{1}$ and sort its values from minimum to maximum. Then we repeat the same process as in the MAR case with the only difference here being that the missing values will be created on $\mathrm{X}_{1}$, and it will be below the cut point if we choose left truncation or above the cut point if we choose right truncation, which in this workflow the right truncation wasn't used. This process is repeated until we reach the desirable proportion of missing values $(5,10,20$, and $30 \%)$.

In the cases of the mixed missingness e.g. MCARMAR-MNAR, the simulation process was done in three steps. If for example we wanted to remove $30 \%$ of the data using this mechanism, in the first step $10 \%$ of the data will be removed using the MCAR method, then the resulting output data will be passed to the MAR mechanism to remove another $10 \%$ and in the end the output data will be passed to the MNAR mechanism in order to remove the last $10 \%$. Always in the mixed missingness mechanisms the missing percentages are equally distributed among MNAR, MAR and MCAR. The reasoning behind this choice was to automatize the whole simulated procedure.

All the functions and code for the simulation process are available on GitHub: (website: https://github.com/ mariekok/impute-metabolomics). Furthermore, the function that is used to simulate the three different missing mechanisms is called simulate_missingness () and it can also been found in the Additional file 1 .

Figure 5 illustrates how missing values in seven different missing mechanisms (MCAR, MAR, MNAR, MCAR-MAR, MCAR-MNAR, MAR-MNAR, MCARMAR-MNAR) affect the distribution of a molecular feature, as exemplified with the case of having $30 \%$ of missing values in the whole dataset.

\section{Imputation methods}

We compared nine imputation methods that have been suggested earlier for metabolomics data-analysis. The methods were divided into three main categories. The first category is the imputation by a single value replacement and includes the mean imputation (MEAN), the minimum observed value, $1 / 2$ minimum ( $1 / 2$ MIN) observed value and zero (ZERO) imputation. The second category is the imputation methods based on local structures and that includes random forest (RF) [28, 29], and k- Nearest Neighbors (KNN) [30-33].The last category contains the imputation methods based on global structures like Singular Value Decomposition (SVD) [34], Probabilistic Principal Component Analysis (PPCA) [7, 13, 35, 36] and Bayesian Principal Component Analysis (BPCA) [36-38]. The comparison of imputation methods was done by using Normalized Root 

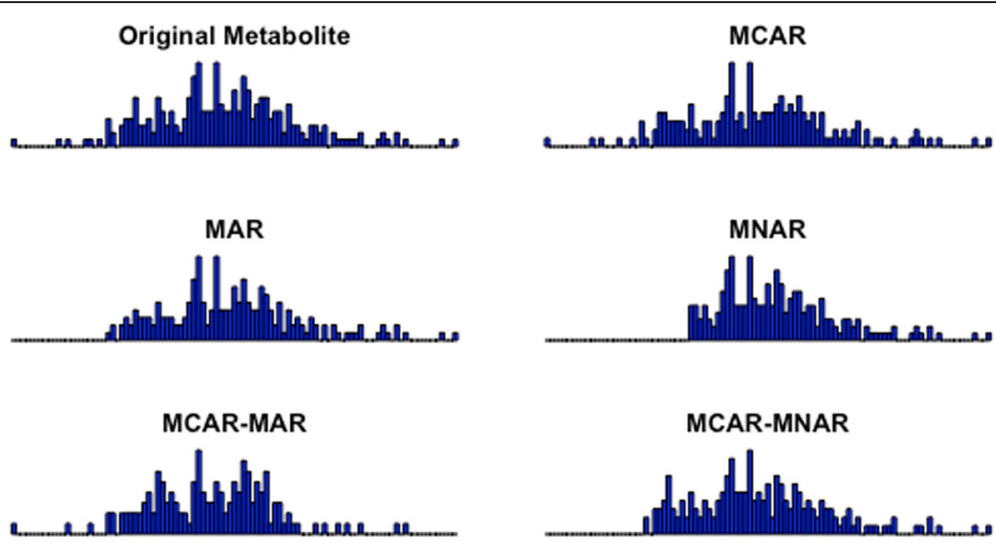

MAR-MNAR

MCAR-MAR-MNAR
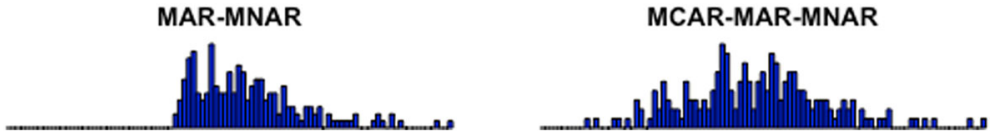

Fig. 5 Distribution based examples of the simulated missing mechanisms. Simulation of missing values for seven different missing mechanisms; Missing Completely At Random (MCAR), Missing At Random (MAR), Missing Not At Random (MNAR), MCAR-MAR, MCAR-MNAR, MNAR-MAR, MNAR-MCAR-MAR for a molecular feature $X$. The range of missing values is set to $30 \%$

Mean Square Error (NRMSE), which has been also earlier suggested for similar purpose [20,39]. NRMSE calculates the difference in the estimation between the imputed value and the original value.

$$
N R M S E=\sqrt{\frac{m e a n\left(\left(X^{\text {comp }}-X^{\text {imp }}\right)^{2}\right)}{\operatorname{var}\left(X^{\text {comp }}\right)}} \text {, where } X^{\text {comp }} \text { is a dataset }
$$

without missing values and $X^{i m p}$ is the same dataset with the missing values being imputed.

\section{Imputation study workflow}

We tested different imputation strategies in real datasets from untargeted LC-MS studies. We simulated MNAR, MCAR, MAR missingness and the different combinations of missingness, namely MCAR-MAR, MCARMNAR, MNAR-MAR, and MNAR-MCAR-MAR, for the evaluation of nine imputation methods (ZERO, MEAN, MIN, $1 \frac{2}{2}$ MIN, SVD, PPCA, BPCA, RF, and KNN) in four different proportions of missing values; $5,10,20$, and $30 \%$. The whole process was repeated 100 times. In each permutation, one dataset out of the 12 was randomly chosen and 200 new molecular features without any missing values were randomly selected. To this subdataset every one of the seven different missing mechanisms were simulated one at a time and with four different percentages of missing values. The new datasets with simulated missing values were used to evaluate the performance of nine imputation methods. The performance of these methods was estimated by calculating NRMSE between the imputed new datasets and the "complete" dataset. A detailed description of the workflow can be seen in Fig. 2.

\section{Supplementary information}

Supplementary information accompanies this paper at https://doi.org/10. 1186/s12859-019-3110-0.

Additional file 1. Contains descriptive statistics measures such as boxplots and correlation plots for the simulated sub-datasets, two summary tables that illustrate the average NRMSEs for all four proportion of missing values for every imputation method and for every type of missingness and one plot illustrating the computational times for every imputation method in every proposition of missing values.

\section{Abbreviations}

$1 / 2$ MIN: Half minimum value; BPCA: Bayesian Principal Component Analysis; BS: Berry Study; KIHD: Kuopio Ischemic Heart Disease Risk Factor Study; KNN: K-Nearest Neighbors; MAR: Missing At Random; MCAR: Missing At Random; MIN: Minimum value; MNAR: Missing Not At Random; PPCA: Probabilistic Principal Component Analysis; RF: Random Forest; SVD: Singular Value Decomposition

\section{Acknowledgments}

The research is part of a research project funded by the Academy of Finland. We would like to thank Dr. Riitta Törrönen from the Institute of Public Health and Clinical Nutrition, UEF, and Riitta Puupponen-Pimiä from VTT for their contribution in the Berry - intervention study.

\section{Authors' contributions}

MK wrote the code for the imputation algorithms, analyzed the results, conducted the simulation studies, and drafted the manuscript. JP wrote the code for the imputation algorithms and supervised the implementation process and critically read and edit the manuscript. KH critically read, edited and participated to the writing of the manuscript. JV and MK collaborated in terms of the human studies, where the data utilized in this manuscripts originates from. All authors read and approved the final manuscript.

\section{Funding}

This study was supported by the Academy of Finland (award number: 2779886) under the Biosciences and Environmental Research (Biotieteiden ja Ympäristön Tutkimuksen) section. Grant recipient was Dr. Kati Hanhineva. The funders do not have any role in the study design, collection, analysis, and interpretation of data. 


\section{Availability of data and materials}

The algorithms were all coded in the R language, version 3.3.1 with the packages: foreach, dplyr, futile.logger, missForest, pcaMethods, doMC, speedglm, imputeLCMD, ggplot2, reshape2, tidyr, matrixStats, VIM. The $\mathrm{R}$ code for imputation functions and R scripts are available in GitHub (https:// github.com/juuussi/impute-metabo). $R$ itself and all packages used are available from CRAN at (http://CRAN.R-project.org/)

The data from the KIHD and BS studies are not publicly available due to sensitive personal information of the study subjects.

\section{Ethics approval and consent to participate}

The KIHD protocol was approved by the Research Ethics Committee of the University of Kuopio, Finland, and complies with Declaration of Helsinki. All the subjects signed a written informed consent. The Berry Study was conducted according to the guidelines laid down in the Declaration of Helsinki and all procedures involving human participants were approved by the Research Ethics Committee of the Hospital District of Northern Savo (Finland). Written informed consent was obtained from all participants.

\section{Consent for publication}

Not applicable.

\section{Competing interests}

The authors declare that they have no competing interests.

\section{Author details}

${ }^{1}$ Institute of Public Health and Clinical Nutrition, University of Eastern Finland, Kuopio Campus, P.O. Box 1627, Fl-70211 Kuopio, Finland. VTT Technical Research Centre of Finland Ltd, P.O. Box 1000, Fl-02044 VIT Espoo, Finland. ${ }^{3}$ Institute of Biomedicine, University of Eastern Finland, Kuopio Campus, P.O. Box 1627, Fl-70211 Kuopio, Finland.

\section{Received: 15 November 2018 Accepted: 20 September 2019} Published online: 11 October 2019

\section{References}

1. Rasmus Madsena, Torbjörn Lundstedt JT. Chemometrics in metabolomics-a review in human disease diagnosis. Anal Chim Acta 2010 ;23-33. Available from: https://ac.els-cdn.com/S0003267009015530/1-s2.0S0003267009015530-main.pdf?_tid=60707e69-7705-41fb-afe0-87d898f915 d8\&acdnat=1533730159_f056b89e1d4955334913ed7f9f5f65bf. [cited 2018 Aug 8].

2. Di Guida R, Engel J, Allwood JW, Weber RJM, Jones MR, Sommer U, et al. Non-targeted UHPLC-MS metabolomic data processing methods: a comparative investigation of normalisation, missing value imputation, transformation and scaling. Metabolomics. 2016;12:93 Available from: http:// www.ncbi.nlm.nih.gov/pubmed/27123000. [cited 2018 Apr 12].

3. Hrydziuszko O, Viant MR. Missing values in mass spectrometry based metabolomics: an undervalued step in the data processing pipeline. Available from: https://link.springer.com/content/pdf/10.1007\%2Fs11306011-0366-4.pdf. [cited 2017 Aug 7].

4. Webb-Robertson BJM, Wiberg HK, Matzke MM, Brown JN, Wang J, JE MD, et al. Review, evaluation, and discussion of the challenges of missing value imputation for mass spectrometry-based label-free global proteomics. J Proteome Res. 2015;14:1993.

5. Armitage EG, Godzien J, Alonso-Herranz V, López-Gonzálvez Á, Barbas C. Missing value imputation strategies for metabolomics data. Electrophoresis. 2015;36(24):3050-60 Available from: http://doi.wiley.com/10.1002/elps.2015 00352. [cited 2017 Oct 24].

6. Gromski PS, Xu Y, Kotze HL, Correa E, Ellis DI, Armitage EG, et al. Influence of missing values substitutes on multivariate analysis of metabolomics data. Metabolites. 2014;4:433-52 Available from: www.mdpi.com/journal/ metabolites/. [cited 2017 May 9].

7. Hrydziuszko O. Development of data processing methods for high resolution mass spectrometry-based metabolomics with an application to human liver transplantation. 2013;Available from: http://etheses.bham.ac. uk/3700/. [cited 2018 Feb 28].

8. Want E, Masson P. Processing and analysis of GC/LC-MS-based metabolomics data: In Humana Press: 2011. p. 277-98. Available from: http://link.springer.com/10.1007/978-1-61737-985-7_17. [cited 2017 Nov 17].
9. Rubin DB. Inference and missing data. Biometrica. 1976;63(3):581-92 Available from: http://qwone.com/ jason/trg/papers/rubin-missing-76.pdf. [cited 2017 Oct 30].

10. Little RJA, Rubin DB. Statistical analysis with missing data [Internet]. second. 2002. Available from: https://leseprobe.buch.de/images-adb/61/97/61976 bf3-cfac-463d-bb88-ca1ddb674cdf.pdf. [cited 2018 Aug 8].

11. Shah JS, Rai SN, Defilippis AP, Hill BG, Bhatnagar A, Brock GN. Distribution based nearest neighbor imputation for truncated high dimensional data with applications to pre-clinical and clinical metabolomics studies. Available from: https://www.ncbi.nlm.nih.gov/pmc/articles/PMC5319174/pdf/12859_2 017_Article_1547.pdf. [cited 2017 May 9]

12. Rhoads $\mathrm{CH}$. Problems with tests of the missingness mechanism in quantitative policy studies. 3(1). Available from: http://cepa.uconn.edu/wpcontent/uploads/sites/399/2015/12/stat-politics-policy2.pdf. [cited 2017 Oct 30].

13. Jamshidian M, Jalal S. Tests of homoscedasticity, normality, and missing completely at random for incomplete multivariate data. Psychometrika. 2010;75:649

14. Hrydziuszko O, Viant MR. Missing values in mass spectrometry based metabolomics: an undervalued step in the data processing pipeline. Metabolomics. 2012;8(S1):161-74 Available from: http://link.springer.com/1 0.1007/s11306-011-0366-4. [cited 2017 Nov 17].

15. Luengo J, García S, Herrera F. On the choice of the best imputation methods for missing values considering three groups of classification methods. Knowl Inf Syst. 2012;32:77-108 Available from: http://sci2s.ugr.es/ sites/default/files/ficherosPublicaciones/1408_2012-luengo-garcia-herreraKAIS.pdf. [cited 2017 Aug 7].

16. Lazar C, Gatto L, Ferro M, Bruley C, Burger T. Accounting for the multiple natures of missing values in label-free quantitative proteomics data sets to compare imputation strategies. J Proteome Res. 2016;15:1116.

17. Schmitt. A comparison of six methods for missing data imputation. J Biomet Biostat. 2015;6 Available from: https://www.omicsonline.org/openaccess/a-comparison-of-six-methods-for-missing-data-imputation-2155-61 80-1000224.pdf. [cited 2017 Nov 17].

18. Taylor SL, Ruhaak LR, Kelly K, Weiss RH, Kim K. Effects of imputation on correlation: implications for analysis of mass spectrometry data from multiple biological matrices. Brief Bioinform. 2017;18(2):312-20 Available from: http://www.ncbi.nlm.nih.gov/pubmed/26896791. [cited 2018 Aug 24].

19. Daniel J. Stekhoven. Nonparametric missing value imputation using random forest. 2016. Available from: https://cran.r-project.org/web/packages/ missForest/missForest.pdf. [cited 2017 Nov 17].

20. Wei R, Wang J, Su M, Jia E, Chen S, Chen T, et al. Missing value imputation approach for mass spectrometry-based metabolomics data. Available from: https://www.nature.com/articles/s41598-017-19120-0.pdf. [cited 2018 Feb 28].

21. Wei R, Wang J, Jia E, Chen T, Ni Y, Jia W. GSimp: A Gibbs sampler based leftcensored missing value imputation approach for metabolomics studies. Nielsen J, editor. PLOS Comput Biol. 2018;14(1):e1005973. Available from: https://doi.org/10.1371/journal.pcbi.1005973. [cited 2018 Oct 22].

22. Rhoads $\mathrm{CH}$. Statistics, politics, and policy problems with tests of the missingness mechanism in quantitative policy studies problems with tests of the missingness mechanism in quantitative policy studies. 2012;3(1) Available from: https://cepa.uconn.edu/wp-content/uploads/sites/399/2 015/12/stat-politics-policy2.pdf. [cited 2018 Oct 23].

23. Salonen JT. Is there a continuing need for longitudinal epidemiologic research? The Kuopio Ischaemic Heart Disease Risk Factor Study. Ann Clin Res. 1988;20(1-2):46-50 Available from: http://www.ncbi.nlm.nih.gov/ pubmed/3408213. [cited 2018 Nov 13]

24. Virtanen JK, Mursu J, Tuomainen T-P, Virtanen HE, Voutilainen S. Egg consumption and risk of incident type 2 diabetes in men: the kuopio ischaemic heart disease risk factor study. Am J Clin Nutr. 2015;101(5):108896 Available from: http://www.ncbi.nlm.nih.gov/pubmed/25832339. [cited 2018 Sep 19]

25. Kolehmainen M, Mykkänen $\mathrm{O}$, Kirjavainen PV, Leppänen T, Moilanen E, Adriaens $M$, et al. Bilberries reduce low-grade inflammation in individuals with features of metabolic syndrome. Mol Nutr Food Res. 2012;56(10):150110 Available from: http://doi.wiley.com/10.1002/mnfr.201200195. [cited 2018 Aug 24].

26. Puupponen-Pimiä R, Seppänen-Laakso T, Kankainen M, Maukonen J, Törrönen $R$, Kolehmainen $M$, et al. Effects of ellagitannin-rich berries on blood lipids, gut microbiota, and urolithin production in human subjects 
with symptoms of metabolic syndrome. Mol Nutr Food Res. 2013;57(12): 2258-63. Available from: http://www.ncbi.nlm.nih.gov/pubmed/23934737. [cited 2018 Nov 13].

27. de Mello VD, Paananen J, Lindström J, Lankinen MA, Shi L, Kuusisto J, et al. Indolepropionic acid and novel lipid metabolites are associated with a lower risk of type 2 diabetes in the finnish diabetes prevention study. sci rep. 2017;7(1):46337 Available from: http://www.ncbinlm.nih.gov/pubmed/2 8397877. [cited 2018 Aug 24].

28. Tang F, Ishwaran H. Random forest missing data algorithms. 2017; Available from: https://arxiv.org/pdf/1701.05305.pdf. [cited 2017 Nov 17].

29. Pantanowitz A, Marwala T. Evaluating the impact of missing data imputation through the use of the random forest algorithm. Available from: https://arxiv.org/ftp/arxiv/papers/0812/0812.2412.pdf. [cited 2017 Nov 17].

30. Batista GEAPA, Monard MC. A study of $k$-nearest neighbour as an imputation method. Available from: http://conteudo.icmc.usp.br/pessoas/ gbatista/files/his2002.pdf. [cited 2017 Nov 17].

31. Beretta L, Santaniello A. Nearest neighbor imputation algorithms: a critical evaluation. BMC Med Inform Decis Mak. 2016;16(Suppl 3):74 Available from: http://www.ncbi.nlm.nih.gov/pubmed/27454392. [cited 2017 Nov 17].

32. Wang P, Tang H, Zhang H, Whiteaker J, Paulovich AG, Mcintosh M. Normalization regarding non-random missing values in high-throughput mass spectrometry data. Pacific Symp Biocomput. 2006;11:315-26.

33. Troyanskaya O, Cantor M, Sherlock G, Brown P, Hastie T, Tibshirani R, et al. Missing value estimation methods for DNA microarrays. BIOINFORMATICS. 2001;17(6):520-5. Available from: https://doi.org/10.1093/bioinformatics/17.6. 520. [cited 2017 Nov 17].

34. Hastie T, Tibshirani R, Sherlock G, Eisen M, Brown P, Botstein D. Imputing missing data for gene expression arrays. 1999; Available from: http://ww. web.stanford.edu/ hastie/Papers/missing.pdf. [cited 2017 Nov 17].

35. Nyamundanda G, Brennan L, Gormley I. Probabilistic principal component analysis for metabolomic data. BMC Bioinformatics. 2010;11(1):571 Available from: http://bmcbioinformatics.biomedcentral.com/articles/10.1186/1471-21 05-11-571. [cited 2017 Nov 17].

36. Stacklies W, Redestig H, Scholz M, Walther D, Selbig J. pcaMethods-a bioconductor package providing PCA methods for incomplete data. 2007: 23(9):1164-1167. Available from: http://www.bioconductor.org. [cited 2017 Aug 9].

37. Bishop CM. Bayesian peA. Available from: http://ai2-s2-pdfs.s3.amazonaws.com/ a24c/135488ef03ea4307ccc650229ac3040be4db.pdf. [cited 2017 Nov 17].

38. Oba S, Sato M-a, Takemasa I, Monden M, Matsubara K-i, Ishii S. A bayesian missing value estimation method for gene expression profile data. Bioinformatics. 2003;19(16):2088-96 Available from: https:/academic.oup. com/bioinformatics/article-lookup/doi/10.1093/bioinformatics/btg287. [cited 2017 Nov 17].

39. Yoon D, Lee E-K, Park T. Robust imputation method for missing values in microarray data. BMC Bioinformatics. 2007:8(Suppl 2)):S6 Available from: http://www.ncbi.nlm.nih.gov/pubmed/17493255. [cited 2017 Nov 17].

\section{Publisher's Note}

Springer Nature remains neutral with regard to jurisdictional claims in published maps and institutional affiliations.

Ready to submit your research? Choose BMC and benefit from:
- fast, convenient online submission
- thorough peer review by experienced researchers in your field
- rapid publication on acceptance
- support for research data, including large and complex data types
- gold Open Access which fosters wider collaboration and increased citations
- maximum visibility for your research: over 100M website views per year
At BMC, research is always in progress.
Learn more biomedcentral.com/submissions

